\title{
Preface for the special issue GandALF 2015
}

\author{
Javier Esparza $^{1} \cdot$ Enrico Tronci $^{2}$
}

Published online: 1 February 2018

(C) Springer-Verlag GmbH Germany, part of Springer Nature 2018

The International Symposium on Games, Automata, Logics and Formal Verification (GandALF) was founded in 2010 by a number of Italian computer scientists interested in mathematical logic, automata theory, game theory, and their applications to the specification, design, and verification of complex systems. Even though the idea of the symposium emerged within the Italian research community, the event has a truly international nature, as witnessed by the composition of the conference committees and the distribution by country of the submitted papers.

The sixth edition of GandALF took place in Genoa, Italy, from September 21 to September 23,2015 . The programme consisted of 13 presentations on algorithmic game theory, automata theory, formal verification, and modal and temporal logics.

This special issue of Acta Informatica contains extended and revised versions of three papers presented at the Symposium. The papers were selected because of their overall quality and the significance of their contributions.

In “Average Energy Games”, Bouyer, Markey, Randour, Larsen and Laursen study games on graphs in which players can lose or gain energy when making a move. The goal of the players is to optimize the long-run average of the accumulated energy. The article studies the complexity of deciding the winner, and proves that memoryless strategies suffice to win.

The paper "Reachability Analysis of Reversal-Bounded Automata on Series-Parallel Graphs", by Dimitrova and Majumdar, introduces an automata model in which a collection of concurrent finite-state machines traverse the edges of a series-parallel graph. The authors prove the decidability and undecidability of variants of the following problem: given an automaton and a graph transformation system generating series-parallel graphs, does the automaton accept some graph generated by the system?

\footnotetext{
Javier Esparza

esparza@in.tum.de

Enrico Tronci

tronci@di.uniroma1.it

1 Faculty of Computer Science, Technische Universität München, Munich, Germany

2 Department of Computer Science, Università di Roma "La Sapienza", Rome, Italy
} 
In "Parameterized Linear Temporal Logics Meet Costs: Still not Costlier than LTL", Zimmermann studies an extension of Linear Temporal Logic (LTL) with costs. The logic allows one to express temporal specifications referring to accumulated costs, like "every request is granted after an execution of cost at most $\$ \mathrm{x} \$$ ". Zimmermann shows that the model checking problem and the problem of solving games with goals expressed in LTL with costs have the same complexity as for standard LTL.

Javier Esparza and Enrico Tronci, PC-chairs of GandALF 2015. 